\title{
Essais d'utilisation d'un rugosimètre à palpeur pour qualifier des surfaces de bois
}

\author{
F. MOTHE \\ INRA, Station de Recherches sur la Qualité des Bois \\ Centre de Recherches de Nancy \\ Champenoux, F 54280 Seichamps
}

\begin{abstract}
Résumé
Un rugosimètre à palpeur, initialement conçu pour la mesure des surfaces métalliques, est décrit. Les différents critères de rugosité délivrés par l'appareil sont présentés.

Des essais de mesures de divers types de surface de bois (bois massif de Merisier raboté ou poncé, planche rabotée de Douglas, placage déroulé de Pin sylvestre, placage tranché de Chêne) ont été effectués avec cet appareil.

On observe une faible dispersion des mesures et une bonne concordance avec l'appréciation visiotactile des mêmes échantillons.
\end{abstract}

Mots clés: Rugosimètre, rugosité du bois, palpeur, critères de rugosité, profil, test visiotactile.

Les expériences décrites ici ont été réalisées à la Station de Recherches sur la Qualité des Bois de l'INRA dans le but de tester un rugosimètre mécanique fonctionnant par palpage nouvellement acquis par le laboratoire.

Le but de cette note technique n'est pas d'apporter des réponses précises aux questions qui se posent en matière de rugosité du bois, mais plutôt de présenter l'appareil, les critères qu'il délivre, et d'étudier de quelle façon cet appareil conçu pour les surfaces métalliques peut être adapté à l'estimation d'une rugosité plus grossière et plus complexe, en l'occurrence celle du bois.

Un appareil de la même marque a déjà été utilisé pour l'estimation de la rugosité de panneaux de particules (GAwronski, 1983).

D'autres rugosimètres fonctionnant sur le même principe ont déjà fourni bon nombre de résultats expérimentaux, dans le cas de bois massif brut de sciage (UsENiUs, 1975) ou poncé (YASUda et al., 1983), de contreplaqués (FAust et al., 1986). La rugosité de panneaux à base de bois, généralement destinés à l'industrie de l'ameublement, a fait l'objet de nombreuses études: Neusser et al., 1971; Lutz, 1964 ; Devantier, 1984 ; Ostman, 1983 ; Russ et al., 1974... On trouvera une bibliographie beaucoup plus complète sur le sujet, et sur la rugosité en général dans les études de Marchal, 1983 ; Triboulot, 1984 \& Mothe, 1985. 
Le nombre de recherches consacrées à la rugosité du bois dans des domaines variés suffit à montrer l'intérêt de la détermination de ce caractère. Un nombre croissant d'appareils est d'ailleurs disponible sur le marché pour mener à bien ces études. L'originalité du rugosimètre Perthen dont il est question ici est de permettre deux niveaux d'interprétation des résultats :

- l'analyse du profil de rugosité permet une étude fine de la surface avec le degré de précision souhaité ;

- lorsqu'un grand nombre d'éprouvettes doit être analysé (étude de variabilité par exemple), des paramètres numériques simples, correspondant à la norme DIN 4768 , sont immédiatement disponibles.

Encore faut-il savoir si ces paramètres - appelés «critères de rugosité " - sont suffisants pour caractériser le profil et, dans laffirmative, lequel de ces critères est le plus adapté à chaque application. Nous apporterons ici des éléments de réponse à cette question.

\section{Description de l'appareil}

Le rugosimètre conçu par la firme Carl Mahr se compose de trois parties :

- une unité de calcul (Perthomètre C50).

— une unité d'avance (PRK), vible).

- deux palpeurs interchangeables RT-250 (2 patins) et FRW-750 ( 1 patin amo-

\subsection{L'unité de calcul}

\subsection{Critères d'évaluation de la surface}

Le profil de surface transmis par le palpeur est dans un premier temps filtré des plus grandes ondulations non imputables à la rugosité, puis divisé par l'unité de calcul en cinq tronçons égaux. Sur chacun d'entre eux sont calculés trois critères locaux de rugosité :

- $\mathrm{R}_{7 \mathrm{i}}$ : l'écart vertical entre le point le plus haut et le point le plus bas de la section du profil $\mathrm{i}$;

- $\mathrm{R}_{\mathrm{pi}}$ : la capacité d'aplanissement qui est l'écart entre le point le plus haut et la ligne moyenne de la section de profil (laquelle est calculée au fur et à mesure du palpage par le rugosimètre) ;

- $\mathrm{R}_{\mathrm{ai}}$ : la rugosité moyenne arithmétique, qui est le rapport de la surface délimitée par le profil et la ligne moyenne à la longueur de la section de profil. profil :

A partir de ces données, quatre critères «globaux » sont calculés sur l'ensemble du

- $R_{z}$ : la rugosité moyenne, qui est la moyenne des cinq $R_{z i}$;

- $R_{\max }$ : la profondeur maximale de rugosité, est égale au plus grand des $R_{z i}$;

- $R_{p}$ : la capacité maximale d'aplanissement, est égale au plus grand des $R_{p i}$;

- $R_{a}$ : la rugosité moyenne arithmétique, est la moyenne des $R_{a i}$. 

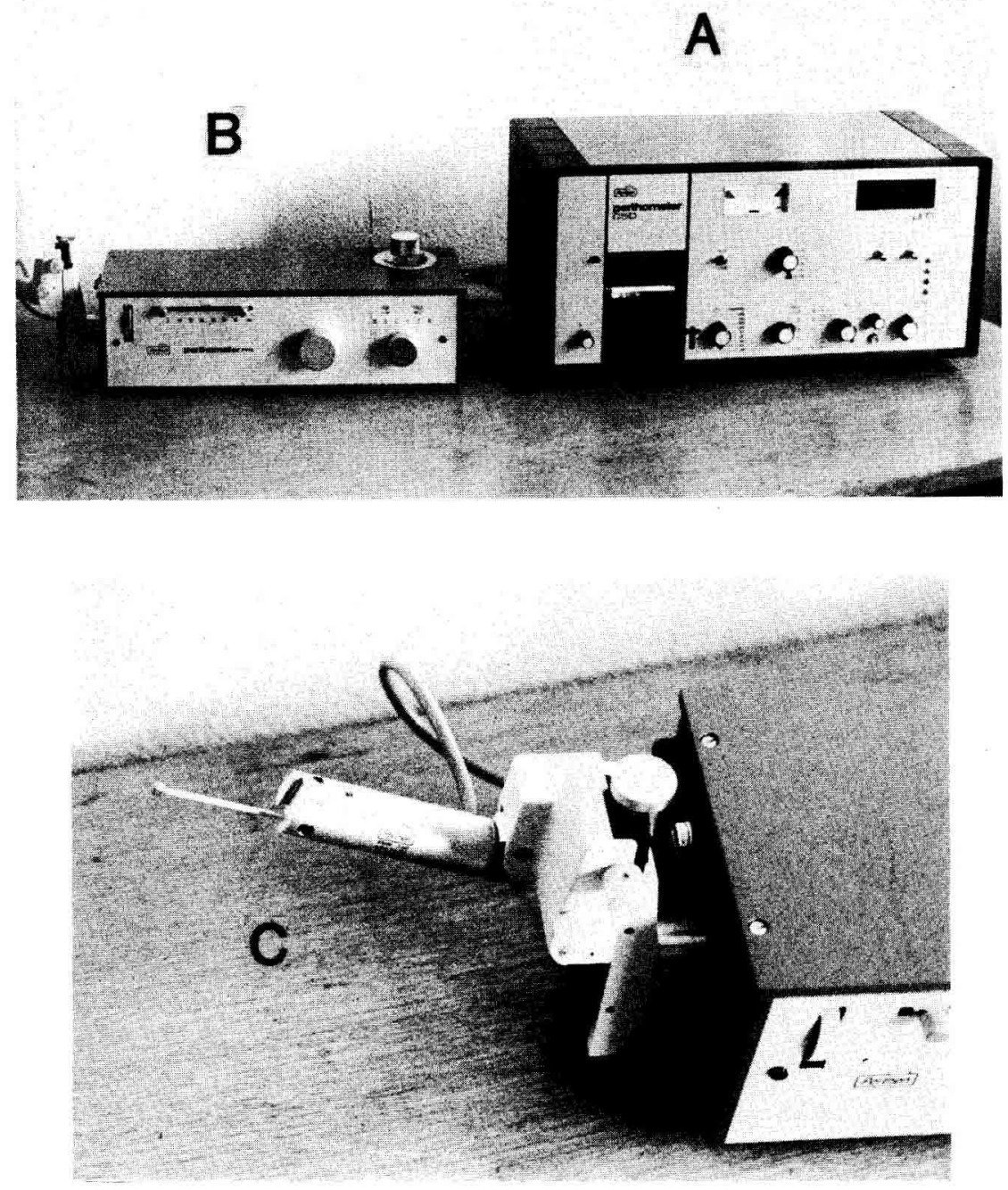

FIG. 1

Le rugosimètre Carl Mahr.

Carl Mahr roughnessmeter.

$\mathrm{A}=$ unité de calcul - computing unit.

$\mathrm{B}=$ unité d'avance - feed unit.

$\mathrm{C}=$ palpeur - stylus. 
Enfin, trois autres critères peuvent être définis sur l'ensemble du profil sans intermédiaire des critères locaux. Ils correspondent tous trois à l'écart entre le point le plus haut et le point le plus bas du profil :

$-R_{1}$ : la rugosité totale, est mesurée sur le profil de rugosité (tout comme les critères précédents), c'est-à-dire un profil filtré des grandes ondulations ;

- $W_{1}$ : l'amplitude des ondulations, est mesurée sur le profil complémentaire du précédent, c'est-à-dire filtré des petites ondulations ;

- $P_{1}$ : l'amplitude totale, est mesurée sur le profil total non filtré.

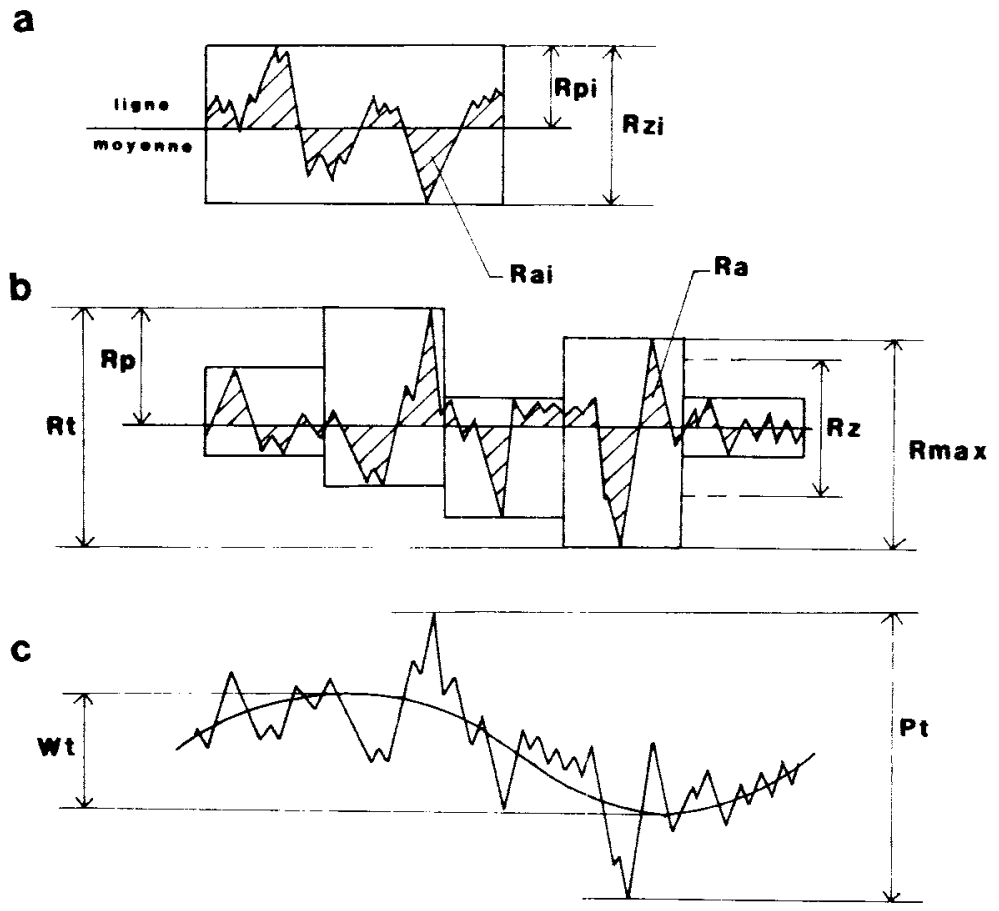

FiG. 2

Critères calculés par le perthomètre C5D.

Criteria delivered by the perthometer C5D.

a) critères de rugosité locaux se rattachant à une section du profil - local roughness criteria related to a section of the profile.

b) critères de rugosité se rattachant à l'ensemble du profil - roughness criteria related to the whole profile.

c) profondeur totale et amplitude des ondulations - total depth and amplitude of undulations.

ligue moyenne - median line 


\subsection{Représentation graphique de la surface}

L'unité de calcul est munie d'une petite imprimante graphique qui permet de tracer les profils filtrés (rugosité ou ondulations) et non filtrés (profil total). Un dispositif de centrage automatique sur le papier facilite la manutention de cette imprimante.

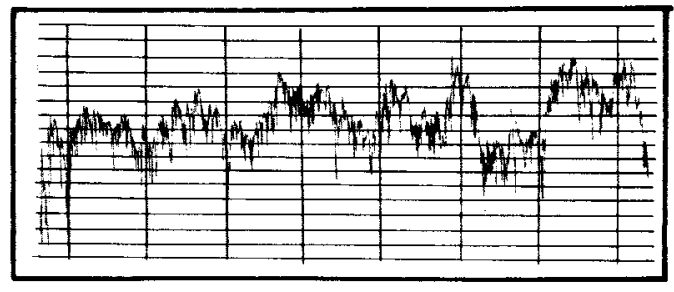

profil total

$$
P_{t}=71,1 \mu \mathrm{m}
$$

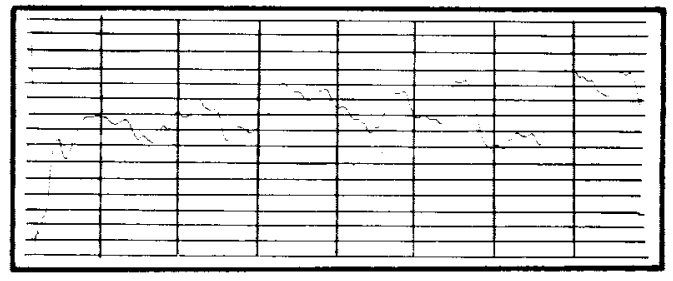

profildes ondulations

$w_{t}=36,1 \mu \mathrm{m}$

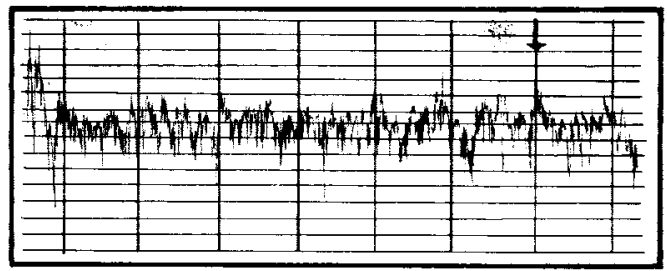

profil de rugosite

$\mathrm{R}_{\mathrm{t}}-61,8 \mu \mathrm{m}$

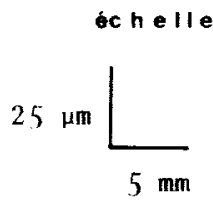

Fic. 3

Profils tracés par limprimante. (L'échantillon utilisé est du Douglas massif raboté - Palpeur standard à deux patins - Longueur du palpage: $48 \mathrm{~mm}$.

Profiles traced by the printer. (The sample is a planed board of Douglas fir - Standard stylus with two skids - Length of measurement : $48 \mathrm{~mm}$ ).

Profil total $=$ whole profile ; profil des ondulations = profile of undulations ; profil de rugosité= roughness profile : échelle = scale. 


\subsection{Restrictions d'utilisation}

La longueur maximale du palpage est de $48 \mathrm{~mm}$, dont seulement $40 \mathrm{~mm}$ interviennent effectivement dans le calcul des critères.

Le seuil de filtrage varie avec la longueur du palpage; deux filtres peuvent être utilisés pour chaque longueur. Ainsi, lorsque celle-ci vaut $48 \mathrm{~mm}$, la limite entre " rugosité » et "grande ondulation" peut être fixée à $8 \mathrm{~mm}$ ou à $2,5 \mathrm{~mm}$ de longueur d'onde (fig. 3).

Il n'est pas possible de tracer les trois profils, ni de déterminer tous les critères cités ci-dessus en un seul palpage. Les cinq valeurs successives de l'un seulement des critères locaux $R_{z i}, R_{p i}$ ou $R_{a i}$ sont affichées un court instant pendant le palpage de la surface. Comme l'unité de calcul n'est munie que d'une seule fenêtre d'affichage, il est nécessaire de choisir, avant la mesure, lequel des critères doit être retenu. Le report de ces valeurs nécessite de plus une attention constante du manipulateur. L'unité de calcul conserve ensuite en mémoire les valeurs de $\mathbf{R}_{z}, \mathbf{R}_{\text {maix }}, \mathbf{R}_{p}, \mathbf{R}_{\mathrm{a}}$, ainsi que de l'un des trois critères $R_{t}, W_{t}$ ou $P_{t}$. Seul le profil correspondant (rugosité, ondulation ou profil total) peut être tracé par l'imprimante. On constate donc que trois palpages sont nécessaires pour connaître l'intégralité des critères. C'est une limitation importante de cet appareil.

\subsection{L'unité d'avance}

Un moteur pas à pas commande le déplacement du palpeur dans la direction horizontale, à la vitesse d'environ $1 \mathrm{~mm} / \mathrm{s}$. Entre le palpeur et l'unité d'avance, un bras articulé permet d'adapter l'appareil à la forme des échantillons les plus divers.

\subsection{Les palpeurs}

Deux palpeurs ont été utilisés dans ces expériences. L'enregistrement des informations (déplacement vertical de la pointe de palpage) s'effectue dans les deux cas par l'intermédiaire d'un circuit inductif. La pointe du palpeur se présente sous la forme d'un cône renversé, dont l'extrémité arrondie présente un diamètre de $10 \mu \mathrm{m}$. Le poids exercé par la pointe sur la surface serait, d'après les constructeurs, de 0,6 g.

\subsection{Palpeur standard RT-250}

Ce palpeur est muni de deux patins, ce qui facilite énormément le réglage de l'appareil, mais présente l'inconvénient de déformer en partie le profil.

La pointe du palpeur RT-250 peut se déplacer verticalement de $250 \mu \mathrm{m}$ de part et d'autre du plan formé par les patins. Cette limite de $500 \mu \mathrm{m}$ est suffisante pour la plupart des surfaces bois ayant subi un traitement de finition, même partiel.

\subsection{Palpeur FRW-750}

Le déplacement vertical de ce palpeur $( \pm 750 \mu \mathrm{m})$ permet son utilisation sur des surfaces brutes de sciage. Il peut fonctionner seul ou avec un patin.

Remarque : un palpeur «macro » est également disponible. La pointe du palpeur FRW-750 est alors remplacée par une bille de diamètre $1.5 \mathrm{~mm}$, qui permet une mesure directe de l'amplitude des ondulations sans filtrage électrique. 


\section{Mesures et résultats}

\subsection{Répétabilité des mesures}

Plusieurs palpages successifs ont été effectués avec le capteur standard sur le même trajet (orthogonal au fil du bois, dans le sens cœur-écorce) et sur trois échantillons :

- une planche rabotée de Douglas (8 passages successifs),

- une planche poncée de Merisier (2 passages), passages).

- un placage de Chêne (épaisseur $0,6 \mathrm{~mm}$ ) collé sur panneau contreplaqué (5

Les critères relevés lors de chaque mesure sont consignés dans le tableau 1 :

Bien que le poids exercé par l'aiguille du palpeur soit très faible, l'influence des passages répétés ne paraît pas négligeable (voir à ce sujet l'étude de Triboulot, 1984).

Dans le cas du Douglas, tous les critères de rugosité voient leur valeur sensiblement réduite entre le premier et le huitième passage. Il est probable que les palpages ont eu pour effet d'araser en partie la surface. On peut cependant s'étonner que l'une des plus fortes différences entre le premier et le dernier enregistrement concerne le critère statistique $R_{a}$ qui devrait être peu affecté par une érosion des crêtes les plus aiguës (voir fig. 4).

Il était en revanche prévisible que le critère $P_{t}$ reste stable puisque les microondulations dues à la rugosité n'interviennent que pour une part dans son évaluation.

Avec le Chêne, essence à bois dur, le phénomène persiste, mais de façon beaucoup moins marquée. Curieusement, la profondeur maximale de rugosité n'est pas affectée par la répétition des passages, sans doute parce que le point le plus haut du profil est situé dans une zone de bois particulièrement dur. Les seules variations importantes concernent les critères $R_{z}$ et $R_{p}$ (ce dernier diminue au cours des 3 premiers passages, puis augmente à nouveau, ce qui est difficilement compréhensible).

On remarquera que seul le premier passage diffère sensiblement des autres pour le Chêne comme pour le Douglas. Les mesures se stabilisent très vite par la suite, comme le montre la figure 4 dans le cas du Douglas.

En ce qui concerne le Merisier, aucune évolution n'est enregistrée entre les deux premiers passages. Il faut peut être attribuer cette particularité à la structure très homogène du bois de cette essence.

D'une façon générale, l'influence du nombre de palpages sur la valeur des critères dans le cas des bois tendres doit être déplorée. En dehors du fait que trois passages sont nécessaires pour obtenir la totalité des critères, il arrive parfois qu'une erreur de lecture ou de manipulation conduise à répéter la mesure. Nous avons vu qu'un écart de 10 p. 100 pouvait être obtenu dans ce cas. Notons cependant qu'il est possible de remédier très simplement à cet inconvénient en déplaçant légèrement le palpeur entre les deux mesures.

\subsection{Comparaison des deux palpeurs}

Lorsque l'échantillonnage présente une forte variabilité, il peut arriver que le palpeur RT-250 arrive à saturation $\left(\mathrm{P}_{\mathrm{t}}>500 \mu \mathrm{m}\right)$. Dans ce cas, le palpage s'interrompt 


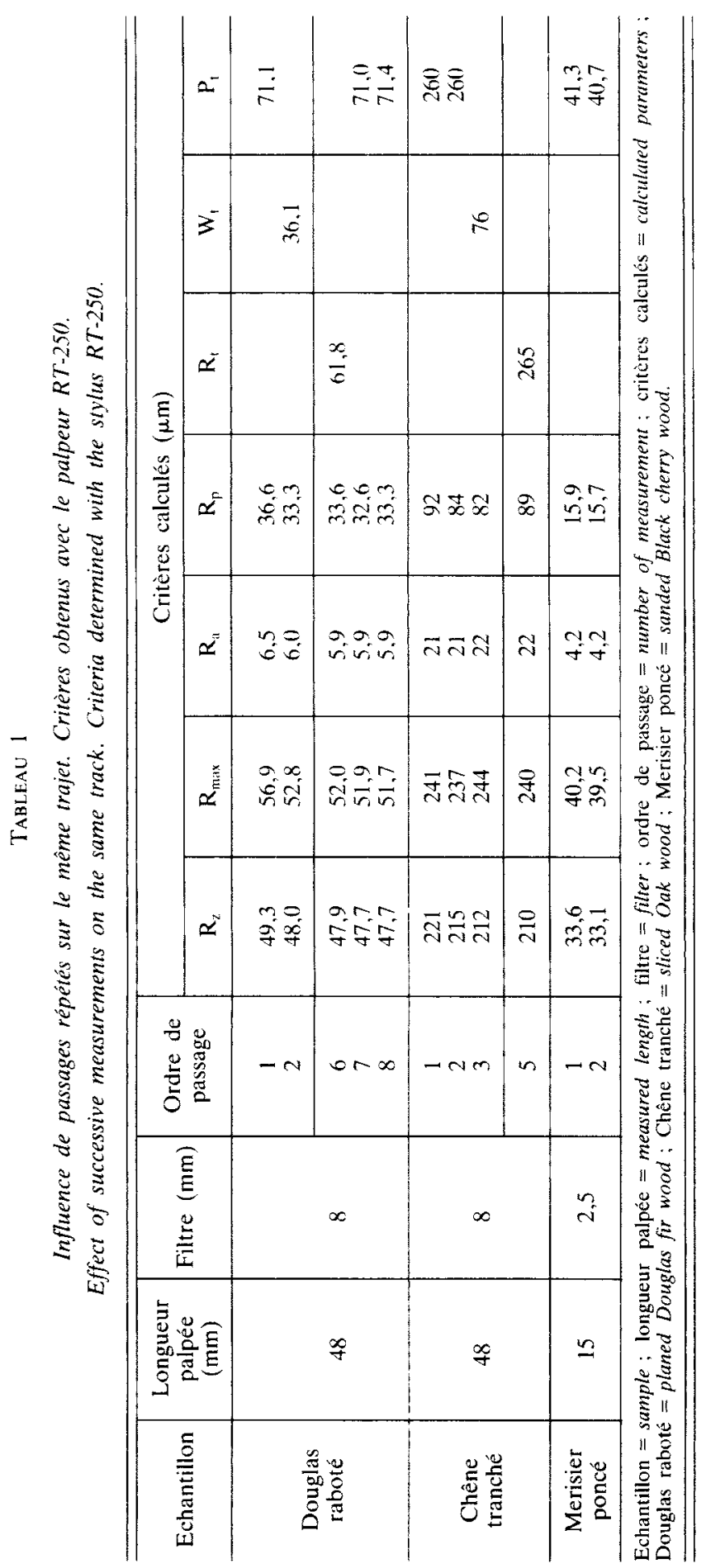




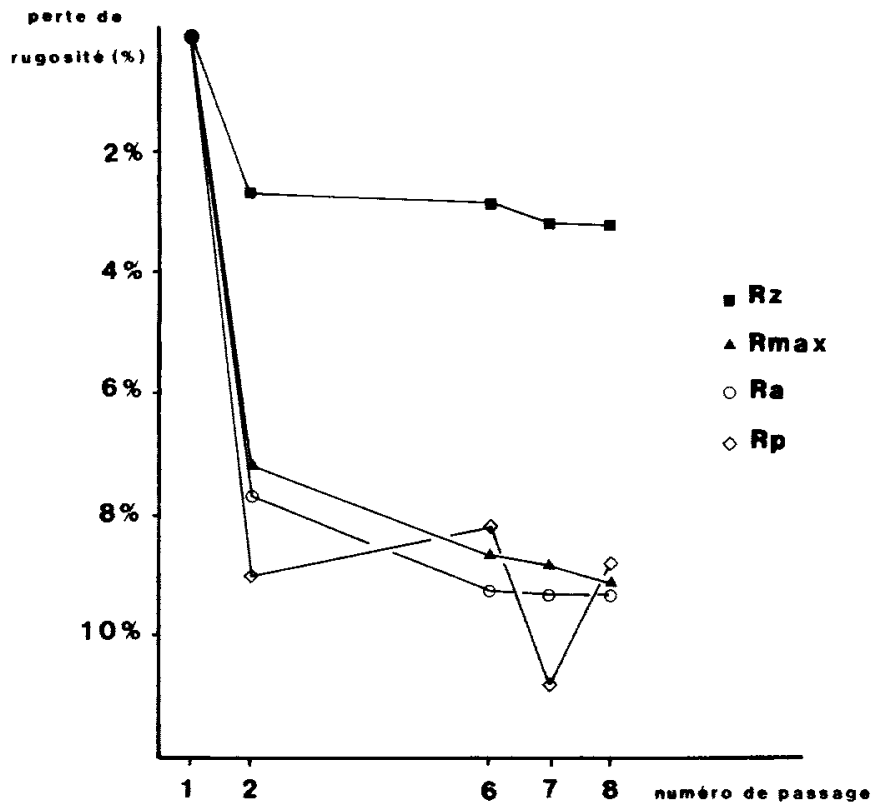

FIG. 4

Perte de rugosité (en p. 100) provoquée par des palpages répétés sur le même trajet.

(L'échantillon est une planche de Douglas rabotée; Palpeur RT-250).

Loss of roughness (in p. 100) induced by successive measurements on the same track.

(The sample is a planed board of Douglas fir; Stylus RT-250).

Perte de rugosité $=$ loss of roughness $;$ numéro de passage $=$ Number of passes .

et un voyant lumineux s'allume. On peut être alors amené à changer de palpeur au cours de l'expérience. Cette démarche n'est concevable que si les résultats obtenus avant et après la substitution sont comparables.

Pour nous en assurer, nous avons testé les deux palpeurs sur la même surface, et approximativement sur le même trajet (longueur : $48 \mathrm{~mm}$, palpage orthogonal au fil du bois).

Deux échantillons ont été utilisés :

- une feuille de placage déroulé de Pin sylvestre, épaisseur $2 \mathrm{~mm}$, non collé ;

- une feuille de placage tranché de Chêne, épaisseur $0,6 \mathrm{~mm}$, non collé.

Le tableau 2 montre les résultats de cette expérience. Ainsi que l'on pouvait s'y attendre, le nombre de patins n'est pas sans effet sur la valeur de la rugosité. Dans le cas du Pin sylvestre cependant, les critères varient peu lorsque l'on utilise un ou deux pảtins, alors que pour le Chêne, le palpeur FRW-750 utilisé avec un seul patin donne des résultats sensiblement différents de ceux obtenus sans patin ou avec 2 patins. 


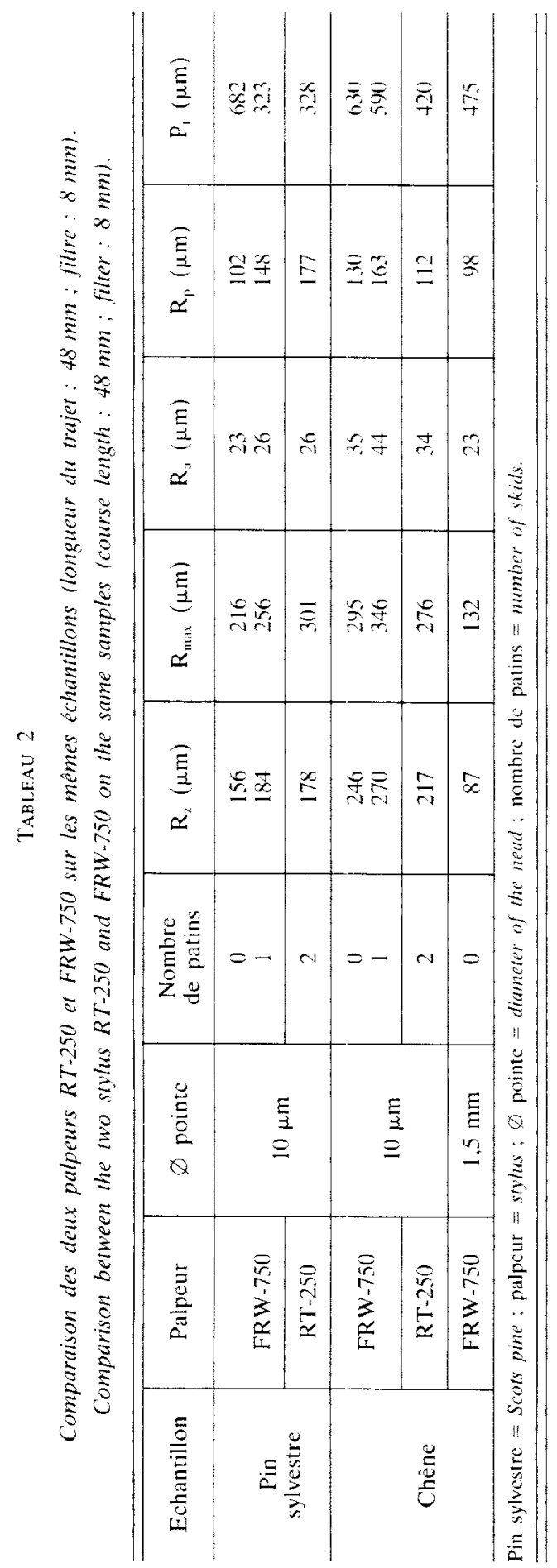


Ce résultat s'explique par le fait que la surface du placage de Pin sylvestre n'est pas exactement parallèle à l'horizontale. La présence d'un ou de deux patins corrige automatiquement l'inclinaison alors que le profil obtenu sans patin est incliné. La différence de cote verticale entre le point le plus haut et le point le plus bas du profil est alors surestimée.

Les critères de rugosité obtenus avec la bille de gros diamètre $(1,5 \mathrm{~mm})$ en guise de palpeur ne sont pas comparables avec les autres valeurs. Il est d'ailleurs impropre de parler dans ce cas de rugosité. Il peut cependant être utile de mesurer ces critères à titre de comparaison avec les résultats des nombreuses études qui ont utilisé un palpeur de type « macro » pour estimer la rugosité du bois (NePveu \& Tran Ngoc, 1984) ou de panneaux de particules (LuTZ, 1964).

$\mathrm{Au}$ vu de ces résultats, il semble que l'utilisation conjointe des palpeurs RT-250 et FRW-750 dans une même expérience soit à déconseiller. En fait, il est probable qu'un autre matériau, plus rigide qu'un placage non collé, aurait donné de meilleurs résultats de ce point de vue.

\subsection{Comparaison de trois types d'usinage}

Trois échantillons de Merisier massif ont été utilisés dans cette expérience :

a) Merisier brut de rabotage,

b) Merisier poncé grossièrement au grain 80 ,

c) Merisier poncé au grain 150 puis patiné à la main.

Ces échantillons avaient préalablement fait l'objet d'un test visiotactile (MotHE, 1985) qui avait conduit au classement suivant, par ordre de rugosité croissante : ponçage fin, rabotage, ponçage grossier.

TABLEAU 3

Comparaison de trois types d'usinage sur du Merisier massif (palpeur RT-250; longueur du trajet: $15 \mathrm{~mm}$; filtre: $2,5 \mathrm{~mm}$ ).

Comparison between three types of machining on solid Black cherry wood (stylus RT-250; course length : $15 \mathrm{~mm}$; filter : $2,5 \mathrm{~mm}$ ).

\begin{tabular}{|c|c|c|c|c|c|}
\hline \multicolumn{2}{|c|}{ Echantillon } & $\mathrm{R}_{\mathrm{z}}(\mu \mathrm{m})$ & $\mathrm{R}_{\max }(\mu \mathrm{m})$ & $\mathbf{R}_{\mathrm{a}}(\mu \mathrm{m})$ & $\mathrm{R}_{\mathrm{p}}(\mu \mathrm{m})$ \\
\hline \multirow[t]{2}{*}{$\begin{array}{l}\text { Ponçage } \\
\text { grossier }\end{array}$} & b & $\begin{array}{l}49 \\
40 \\
53\end{array}$ & $\begin{array}{l}59 \\
48 \\
63\end{array}$ & $\begin{array}{l}8,5 \\
7,8 \\
9,2\end{array}$ & $\begin{array}{l}33 \\
24 \\
29\end{array}$ \\
\hline & moy. & 47 & 57 & 8,5 & 29 \\
\hline \multirow{2}{*}{ Rabotage } & & $\begin{array}{l}45 \\
40\end{array}$ & $\begin{array}{l}40 \\
49\end{array}$ & $\begin{array}{l}5,2 \\
5,1\end{array}$ & $\begin{array}{l}22 \\
18\end{array}$ \\
\hline & moy. & 43 & 51 & 5,1 & 20 \\
\hline \multirow[t]{2}{*}{$\begin{array}{c}\text { Ponçage } \\
\text { fin }\end{array}$} & $\mathrm{c}$ & $\begin{array}{l}34 \\
33 \\
34\end{array}$ & $\begin{array}{l}40 \\
39 \\
36\end{array}$ & $\begin{array}{l}4,2 \\
4,3 \\
3,9\end{array}$ & $\begin{array}{l}16 \\
16 \\
11\end{array}$ \\
\hline & moy. & 34 & 39 & 4,1 & 14 \\
\hline
\end{tabular}

Ponçage grossier $=$ rough sanding $;$ rabotage $=$ planing $;$ ponçage fin $=$ fine sanding $;$ moy.$=$ mean . 
Le tableau 3 présente les résultats que nous avons obtenus avec le palpeur RT-250 à deux patins. La distance parcourue était de $15 \mathrm{~mm}$ dans le sens orthogonal au fil du bois. Les passages successifs se rapportent ici a des emplacements différents sur la surface.

Les critères calculés par le rugosimètre confirment entièrement les impressions tactiles. On peut toutefois remarquer que les critères $R_{z}$ et $R_{\text {max }}$ se différencient en ce sens qu'ils classent l'échantillon a) plus proche de b) que de c), alors que l'inverse se produit pour les autres critères.

\subsection{Analyse fine de deux placages de chêne}

Nous avons utilisé deux placages de chêne (épaisseur $0,6 \mathrm{~mm}$, tranchage sur dosse) collés sur support contreplaqué et d'aspects visiotactiles très différents. Des mesures effectuées à l'aide d’un rugosimètre pneumatique avaient confirmé ce résultat. Nous noterons (1) l'échantillon le plus rugueux, et (2) le plus lisse.

Les mesures ont été effectuées avec le palpeur RT-250 à deux patins, dans le sens orthogonal au fil du bois.

L'amplitude totale $P_{\text {}}$ présente ici peu d'intérêt puisqu'elle mesure surtout l'importance des déformations provoquées par le collage. Elle permet toutefois de vérifier que le déplacement vertical du palpeur n’a pas atteint la valeur critique de $500 \mu \mathrm{m}$.

\section{TABLEAU 4}

Analyse de deux placages de Chêne (palpeur RT-250; longueur du trajet: $48 \mathrm{~mm}$; filtre : $8 \mathrm{~mm}$ ).

Measurements on two Oak veneers (stylus RT-250; course length: $48 \mathrm{~mm}$; filter : $8 \mathrm{~mm}$ ).

\begin{tabular}{|c|c|c|c|c|c|c|}
\hline Echantillon & Trajet & $\mathrm{R}_{2}(\mu \mathrm{m})$ & $\mathrm{R}_{\max }(\mu \mathrm{m})$ & $\mathrm{R}_{\mathrm{at}}(\mu \mathrm{m})$ & $R_{p}(\mu m)$ & $P_{t}(\mu m)$ \\
\hline \multirow[t]{2}{*}{$\begin{array}{l}\text { jugé } \\
\text { « rugucux » }\end{array}$} & $\begin{array}{l}a \\
b \\
c \\
d \\
e\end{array}$ & $\begin{array}{l}221 \\
143 \\
154 \\
151 \\
187\end{array}$ & $\begin{array}{l}241 \\
166 \\
189 \\
241 \\
226\end{array}$ & $\begin{array}{l}21 \\
17 \\
15 \\
14 \\
21\end{array}$ & $\begin{array}{l}92 \\
58 \\
55 \\
80 \\
95\end{array}$ & $\begin{array}{r}260 \\
98 \\
192 \\
221 \\
211\end{array}$ \\
\hline & moyenne & 171 & 213 & 18 & 76 & 196 \\
\hline \multirow[t]{2}{*}{$\begin{array}{l}(2) \\
\text { jugé } \\
\text { «lisse » }\end{array}$} & $\begin{array}{l}a \\
b \\
c \\
d \\
e\end{array}$ & $\begin{array}{r}98 \\
113 \\
150 \\
97 \\
111\end{array}$ & $\begin{array}{l}184 \\
189 \\
226 \\
234 \\
172\end{array}$ & $\begin{array}{r}7 \\
9 \\
18 \\
9 \\
11\end{array}$ & $\begin{array}{l}37 \\
46 \\
71 \\
61 \\
50\end{array}$ & $\begin{array}{l}176 \\
177 \\
235 \\
211 \\
170\end{array}$ \\
\hline & moyenne & 114 & 201 & 11 & 53 & 194 \\
\hline \multicolumn{2}{|c|}{$\begin{array}{l}\text { Comparaison des moyen- } \\
\text { nes. Valeur et seuil de si- } \\
\text { gnification du test } t\end{array}$} & $\underset{*}{3,29}$ & $\begin{array}{l}0,60 \\
\text { NS }\end{array}$ & $\begin{array}{c}2,82 \\
*\end{array}$ & $\begin{array}{c}2,24 \\
\text { NS }\end{array}$ & $\begin{array}{c}0,09 \\
\text { NS }\end{array}$ \\
\hline
\end{tabular}

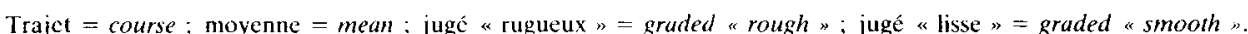
Comparaison des moyennes. Valeur et seuil de signification du test $\mathrm{t}=$ Comparison of means. Value and significance level of $t$ test.

NS : non significatif $=$ not significam

* Significatif au scuil de $5 \mathrm{p}$. $1(00$ (8 degrés de liberté) = significant at 5 p. 100 level ( 8 degrees of frédom). 
Au vu du tableau 4 , les critères $R_{\text {max }}$ et $R_{p}$ ne paraissent pas en mesure de distinguer les deux surfaces. Ces critères ont en commun la particularité d'être fortement influencés par un défaut localisé : profondeur d'un vaisseau (pour $\mathbf{R}_{\max }$ ), crête particulièrement accentuée. Or, à l'examen visiotactile des placages - qui ne permet pas de détecter la profondeur des vaisseaux - il semble que ce soit plutôt la fréquence des zones poreuses qui soit responsable de la différence de rugosité ; l'échantillon (1) présente en effet des cernes beaucoup plus fins que le (2).

Si lon admet que tous les palpages recouvraient à la fois des zones de vaisseaux et des zones de fibres, on comprend que les critères $R_{z}$ et $R_{a}$ qui tiennent compte de l'ensemble du profil, soient les plus aptes à distinguer les deux échantillons. Les résultats fournis par ces deux critères sont effectivement cohérents avec les tests pneumatiques et visiotactiles.

Ces derniers nous fournissent cependant une indication supplémentaire : la différence de rugosité entre les placages (1) et (2) persiste lorsque l'on ne considère que les zones dépourvues de vaisseaux. Nous avons voulu vérifier ce phénomène en limitant le trajet du palpeur à une zone homogène.

La différence de rugosité entre les placages (1) et (2) apparaît en effet beaucoup plus nettement dans les zones de fibres que dans les zones de vaisseaux (tabl. 5). Seul le critère $R_{p}$ offre des performances comparables dans les deux zones. Ce point mériterait d'être étudié plus précisément; il est possible que l'explication réside dans le fait que $R_{p}$ est peu influencée par la taille des vaisseaux.

\section{TABLEAU 5}

Analyse de deux placages de Chêne. Palpage effectué dans une zone homogène de bois final ou de bois initial (palpeur RT-250; longueur du trajet: $15 \mathrm{~mm}$; filtre : $2,5 \mathrm{~mm}$ ).

Measurements on two Oak veneers. Measurements made in homogeneous zone of late wood or early wood (stylus RT-250; course length: $15 \mathrm{~mm} ;$ filter : $2.5 \mathrm{~mm}$ ).

\begin{tabular}{|c|c|c|c|c|c|c|}
\hline \multicolumn{2}{|c|}{ Echantillon } & $\mathrm{R}_{z}(\mu \mathrm{m})$ & $\mathrm{R}_{\max }(\mu \mathrm{m})$ & $\mathrm{R}_{\mathrm{a}}(\mu \mathrm{m})$ & $\mathrm{R}_{\mathrm{r}}(\mu \mathrm{m})$ & $P_{1}(\mu \mathrm{m})$ \\
\hline \multirow{2}{*}{$\begin{array}{c}(1) \\
\text { rugueux }\end{array}$} & bois final & $\begin{array}{l}75 \\
53\end{array}$ & $\begin{array}{l}86 \\
61\end{array}$ & $\begin{array}{r}10 \\
7\end{array}$ & $\begin{array}{l}43 \\
31\end{array}$ & $\begin{array}{r}102 \\
96\end{array}$ \\
\hline & bois initial & $\begin{array}{l}156 \\
160\end{array}$ & $\begin{array}{l}193 \\
178\end{array}$ & $\begin{array}{l}27 \\
25\end{array}$ & $\begin{array}{l}85 \\
78\end{array}$ & $\begin{array}{l}246 \\
274\end{array}$ \\
\hline \multirow{2}{*}{$\begin{array}{l}(2) \\
\text { lisse }\end{array}$} & bois final & $\begin{array}{l}42 \\
44\end{array}$ & $\begin{array}{l}52 \\
51\end{array}$ & $\begin{array}{l}5 \\
5\end{array}$ & $\begin{array}{l}18 \\
24\end{array}$ & $\begin{array}{l}67 \\
67\end{array}$ \\
\hline & bois initial & $\begin{array}{l}135 \\
166\end{array}$ & $\begin{array}{l}160 \\
183\end{array}$ & $\begin{array}{l}19 \\
27\end{array}$ & $\begin{array}{l}53 \\
57\end{array}$ & $\begin{array}{l}149 \\
175\end{array}$ \\
\hline $\begin{array}{l}\text { Rapport } \\
(1) /(2)\end{array}$ & $\begin{array}{l}\text { bois final } \\
\text { bois initial }\end{array}$ & $\begin{array}{l}1.5 \\
1.0\end{array}$ & $\begin{array}{l}1,4 \\
1,1\end{array}$ & $\begin{array}{l}1,6 \\
1,1\end{array}$ & $\begin{array}{l}1,7 \\
1,5\end{array}$ & $\begin{array}{l}1,5 \\
1,6\end{array}$ \\
\hline
\end{tabular}

Rugueux $=$ rough $;$ lisse $=3$ mooth $;$ rapport $=$ ratio $;$ bois final $=l a t e$ wood $;$ bois initial $=$ early wood .

Quoi qu'il en soit, il n'est plus possible, au vu de ces résultats, d'attribuer à la seule largeur des cernes ou à leur fréquence la différence de rugosité entre les deux échantillons. Cette expérience ne nous permet cependant pas de savoir si la rugosité "intrinsèque " des zones de fibres n'est pas liée mécaniquement ou génétiquement à la largeur des cernes. 


\section{Conclusion - Perspectives d'utilisation}

Les quelques expériences dont nous avons rapporté les résultats ici nous semblent bien augurer des possibilités de l'appareil en matière de recherche sur la rugosité du bois.

Quelques défauts doivent cependant être soulignés :

- Comme tous les appareils fonctionnant par palpage, ce rugosimètre n'offre qu'une analyse bidimensionnelle de la surface. Un appareillage relativement simple permettrait de tenir compte de la troisième dimension en multipliant les profils parallèles (ou croisés) sur la même surface (Triboulot, 1984, op. cit.).

- Le contact avec le palpeur provoque indubitablement une légère érosion de la surface, notamment dans le cas des bois tendres. Ces dommages, même s'ils ne sont pas discernables à la vue ou au toucher, sont détectés par le rugosimètre lui-même. lors d'un deuxième passage sur le même trajet.

- Il est difficile de comparer les valeurs de rugosité données par le palpeur standard à deux patins avec celles obtenues avec le palpeur de grande capacité, qui ne possède qu'un patin. La même remarque vaut probablement pour deux mesures effectuées avec des intensités de filtrage différentes. Pourtant, étant donné la conception de l'appareil, le changement de filtre est souvent obligatoire lorsque l'on modifie la longueur de palpage.

- Les critères de rugosité calculés par le rugosimètre apparaissent un par un sur l'affichage digital. Le report de ces valeurs est une source d'erreur et une perte de temps. De plus, l'ensemble des critères ne peut ètre obtenu qu'en effectuant trois passages successifs sur le même échantillon.

Il serait facile de remédier aux deux derniers inconvénients en adjoignant à l'unité de calcul actuelle un micro-ordinateur et une interface lui permettant de saisir les données brutes. Le profil pourrait alors être manipulé à sa convenance par lopérateur afin de tenir compte du nombre de patins utilisés ou de permettre le calcul et l'enregistrement de tous les critères désirés.

D’ores et déjà, le rugosimètre Mahr devrait pouvoir être exploité utilement dáns sa version actuelle, ne serait-ce qu'en substitution des appréciations visiotactiles. Nous avons déjà vérifié la concordance entre les deux méthodes lorsque le contraste de rugosité est important. Il serait intéressant de poursuivre cette comparaison sur un plus grand nombre d'échantillons, et peut-être d'en déduire quel critère (ou quelle combinaison de critères) est le plus approprié pour rendre compte de la sensation de rugosité. Ce résultat permettrait par exemple la comparaison objective de différents modes de finition pour lesquels l'appréciation visiotactile est prépondérante.

Une démarche similaire pourrait être employée pour permettre la prédiction de la quantité de vernis à employer, ou encore de la perte de matière au ponçage au vu du matériau brut de tranchage ou de rabotage.

Cet appareil devrait faciliter également les recherches visant à établir des liens entre les propriétés physiques ou anatomiques du bois et la qualité des surfaces obtenues. 


\section{Summary \\ Testing of a roughness-meter for assessment of wood surfaces}

A roughness-meter with stylus designed for metallic surfaces is described. The various parameters determined by the apparatus are presented.

Various tests were made with different types of wood surfaces (planed and sanded solid wood of Black Cherry, planed boards of Douglas fir, peeled veneer of Scots pine, sliced veneer of Oak).

A small measurements dispersion can be observed in good agreement with visual and tactile tests.

Key words : Roughness-meter, roughness of wood surface, stylus, roughness parameters, profile, visual and tactile tests.

\section{Zusammenfassung}

\section{Bestimmung der qualitat von holzoberflachen mit einem rauhigkeits-messinstrument}

Ein Rauhigkeitsmessgerät urprünglich gedacht für Messungen von Metalloberflächen, wird beschrieben. Die verschiedenen Rauhigkeitsparameters, die der Apparat bestimmt, werdern erklärt.

Messversuche an verschiedenen Typen von Holzoberflächen (gehobeltes und geschliffenes Massivholz von Kirschbaum, geschliffenes Brett von Douglasie, Schälfurnier von Kiefer und Messerfurnier von Eiche) wurden mit diesem Apparat durchgeführt.

Einige Angaben sind für folgende Aspekte gegeben : Wiederholbarkeit der Messungen,. Wirkung des Types des Me stiftes, Wirkung der Verarbeitung, Vergleich mit den Seh-Tastmessungen und Wirkung der anatomischen Charakteren des Holzes.

\section{Références bibliographiques}

Devantier B., 1984. Automatische Messplatz zur Bestimmung der Oberflächenunruhe von endbehandelten Möbeloberflächen. Holztechnologie, 25 (2), 67-68.

FAust T.D., JAMES T.R., 1986. Characterizing the roughness of southern pine veneer surfaces. Forest Products Journal, 36 (11/12), 75-81.

Gawronski A., URBanik E., 1983. Wplyw rozcieňczalników organicznych na zmiany chropowatošci powierzchni plyt wiórowych (Effect of organic solvents on the surface roughness of particleboard). Prace Inst. Tech., Drewna (Pologne), 29 (1-2), 41-42. (résumé dans FPA 1984, 7 (6), n"1665).

Lutz P., 1964. Contribution à l'appréciation de l'état de surface des panneaux de particules. Document Agglos 490, Etude $n^{\circ} 48$ du Centre Technique du Bois, 67 p.

Marchal R., 1983. Intérêt de la prise en compte de caractéristiques physiques et anatomiques simples du bois de chêne pour l'appréciation de la qualité des placages d'ébénisterie. D.E.A. «Sciences du Bois», Université de Nancy I, octobre 1985.

Mothe F., 1985. Essai et comparaison de trois méthodes de classement de surface de bois massif pour leur rugosité : méthodes pneumatique et sensorielles. Ann. Sci. For., 42 (4), 435-452. 
Nepveu G., Tran Ngoc T., 1984. Relations entre les composantes densitométriques et l'état de surface de planches rabotées chez Pseudotsuga menziesii. Ann. Sci. For., 41 (2), 171-194.

Neusser H., Krames U., 1971. Die Oberflächengestalt von Holzspanplatten. Ihr Erfassung und ihre Schönheitswirkung. Holz als Roin und Werkstoff, 29 (3), 103-118.

Ostman B.A.L., 1983. Surface roughness of wood based panels after aging. Forest Products Journal., 33 (7-8), 35-42.

Russ W., Zumpe W., 1974. Zur Bewertung der Oberflächengüte von Möbeln. Holztechnologie, 15 (4), 234-239.

Triboulot P.. 1984. Réflexions sur les surfaces et mesures des états de surface du bois. Ann. Sci. For. 41 (3), 335-354.

Usenius A., 1975. Surface smoothness measurement of sawn timber. Paperi ja Puu, 12, $871-875$.

YASUdA A., SADOH T., NAKAto K.. 1983. Visual and tactile roughness of hardwood surfaces relating to physical roughness. Mokuzai Gakkaishi. 29 (11), 731-737. 